Meta

Journal des traducteurs

Translators' Journal

\title{
Du sens au signe : La néologie des langues africaines en contexte terminologique
}

\section{Nazam Halaoui}

Volume 38, numéro 4, décembre 1993

Le Je du traducteur

The $I$ of the Translator

URI : https://id.erudit.org/iderudit/002997ar

DOI : https://doi.org/10.7202/002997ar

Aller au sommaire du numéro

Éditeur(s)

Les Presses de l'Université de Montréal

ISSN

0026-0452 (imprimé)

Découvrir la revue

Citer cet article

Halaoui, N. (1993). Du sens au signe : La néologie des langues africaines en contexte terminologique. Meta, 38(4), 704-718. https://doi.org/10.7202/002997ar d'utilisation que vous pouvez consulter en ligne. 


\section{DU SENS AU SIGNE. LA NÉOLOGIE DES LANGUES AFRICAINES EN CONTEXTE TERMINOLOGIQUE}

\section{INTRODUCTION}

Nombreuses sont les langues africaines qui sont aujourd'hui utilisées dans les processus éducatifs liés au développement. Il en est ainsi, pour ne retenir qu'un domaine d'utilisation parmi les plus importants, en alphabétisation, initiale certes, mais aussi et surtout en post-alphabétisation, où est privilégiée la vulgarisation de la connaissance en matière de gestion, d'agriculture, de santé, etc. Si la langue de l'individu cible de l'éducation est l'instrument de communication le meilleur pour transmettre la connaissance éducative, cette langue doit être en mesure d'assumer la fonction qui lui est ainsi assignée, ce qui n'est pas toujours le cas dans les spécialités retenues par l'éducation pour le développement. En effet, la connaissance à transmettre est, dans le cas général, étrangère au milieu naturel d'utilisation des langues africaines, elle y est introduite par l'éducation, ce qui explique que ces langues ne la prennent pas en charge.

De ce fait, qu'on choisisse la voie de la traduction d'une langue étrangère ou celle de la rédaction en langue africaine, car ces deux voies sont aujourd'hui suivies, l'élaboration des ouvrages consignant une connaissance spécialisée requiert la confection de terminologies relatives aux spécialités retenues. On observe ainsi une activité terminologique intense, même si celle-ci ne bénéficie pas toujours de l'organisation et des compétences qui lui sont dues, activité dans laquelle s'inscrit nécessairement un travail néologique, mené dans le but de combler les lacunes que montrent les langues africaines dans leur prise en charge de la communication dans les domaines spécialisés. Ainsi, si l'objet de la terminologie est de mettre à la disposition des intéressés les ressources de la langue, celui de la néologie est de compléter celles-ci, afin que, dans une spécialité donnée, le vocabulaire montre une adéquation certaine avec la réalité.

Se situant résolument dans un contexte terminologique, le présent article se propose, d'une part, de préciser ce contexte, à travers les données à prendre en compte par le terminologue et les voies qui s'offrent à lui, d'autre part, de faire ressortir l'ensemble des procédés de néologie reconnus dans les langues africaines en présentant les faits selon une perspective à la fois morphologique et néologique, enfin, de tenir compte, dans cette présentation, des processus en œuvre, en privilégiant plutôt l'aspect néographique, qui est 
propre à la pratique du terminologue, que l'aspect néologique. Pour trois raisons bien précises, les exemples en langue africaine donnés ici sont pris dans la seule langue bambara. En premier lieu, la synthèse qui est proposée dans ce texte prend sa source dans l'observation de nombreuses langues africaines ${ }^{1}$ autres que le bambara. En second lieu, il s'agit de présenter les procédés en usage, et la langue bambara est alors tout à fait indiquée 2 , et non de prouver l'existence de ceux-ci dans les langues africaines. Enfin, on sait que le cadre attribué, dans une revue, à un tel article ne permet jamais de s'étendre sur les exemples.

\section{TERMINOLOGIE ET NÉOLOGIE}

En terminologie des langues africaines, compte tenu des capacités des langues en présence et des langues étrangères avec lesquelles celles-ci sont en contact, l'enquête est double. Elle est une enquête ethnographique et est alors effectuée dans la langue africaine dans laquelle la terminologie est en élaboration. Elle est aussi une enquête savante, et est alors menée dans une langue étrangère, prise comme langue modèle en raison de son aptitude reconnue à prendre en charge la communication dans le domaine spécialisé en étude, et en fonction de laquelle la terminologie en langue africaine sera confectionnée (Halaoui 1991b: 5).

Une telle enquête doit donner à l'enquêteur, d'une part, les terminologies du domaine retenu dans les deux langues ci-dessus mentionnées, et, d'autre part, une bonne connaissance des réalités des milieux africain et étranger relatifs au domaine en question. La même enquête doit permettre à l'enquêteur de comparer les terminologies en présence, afin de faire ressortir les capacités et les lacunes de la terminologie en langue africaine, ceci, dans le but de retenir les unes et de combler les autres. Au terme d'une telle enquête, le terminologue africaniste, qui est en possession des terminologies et de la connaissance des réalités, se trouve, dans le cas général, en présence d'un certain nombre de signifiés particuliers.

Ces signifiés sont relatifs au domaine africain de la spécialité en question. Ils sont parvenus au terminologue à travers l'observation systématique qu'il a faite du milieu africain, ou lui sont suggérés par la terminologie correspondante offerte par la langue modèle où ils sont exprimés. Enfin, fait le plus important en ce qui concerne la néologie, ces signifiés ne sont pas pris en compte par la terminologie de la spécialité en étude disponible dans la langue africaine. Tel est, en terminologie des langues africaines, le contexte qui apparaît à la base de la mise en ouvre de l'activité néologique (Halaoui $1989: 10-15)$.

En effet, si la langue africaine concernée doit, de manière effective, et au sein de la spécialité considérée, assumer une fonction de communication et, donc, si la terminologie qui est en élaboration, l'est dans la perspective d'être effectivement utilisée dans le domaine spécialisé considéré, les signifiés en présence doivent, par attribution à chacun d'eux d'un signifiant, pouvoir constituer des signes, qui entreront dans la langue, deviendront des termes techniques, et seront incorporés à la nomenclature de la terminologie en question. Intégrer ces signifiés à la langue africaine, c'est certes créer des signes qui les prendront en charge, c'est aussi et surtout disposer de signifiants aptes à la fois à exprimer ces signifiés et à constituer avec eux des signes.

Au terminologue africaniste qui, pendant l'élaboration de la terminologie d'une spécialité donnée, se trouve en possession de tels signifiés, se pose donc un problème, celui de l'obtention des signifiants correspondants. Un signifiant, dans une langue donnée, n'apparaissant pas seul, mais associé à un signifié au sein d'un signe, la recherche des signifiants sera d'abord une recherche de signes exprimant effectivement, ou montrant une aptitude à exprimer, les signifiés en possession du terminologue. Cette recherche se 
fera bien sûr dans l'une des langues concernées par l'élaboration de la terminologie, c'est-à-dire, soit la langue africaine dans laquelle la terminologie est en élaboration, soit la langue modèle en fonction de laquelle cette même terminologie est en confection, soit, enfin, une autre langue africaine, en contact avec la première et montrant avec elle des échanges lexicaux.

Pour pouvoir être exprimé, un signifié peut requérir son association au signifiant d'un seul signe, ou aux signifiants de plusieurs signes, de l'une de ces langues. Il y a là une réalité qui oriente le terminologue dans deux voies différentes de son activité néologique. En effet, quand le signifié demande son association au signifiant d'un seul signe, le terminologue pourra, selon les contraintes du moment, adopter ce signe ou l'ignorer. Quand, par contre, le même signifié demande son association aux signifiants de plusieurs signes, ou quand un signe unique, reconnu apte, est volontairement ignoré par le terminologue, celui-ci devra nécessairement créer le nouveau signe, dont le signifiant pourra véhiculer notre signifié.

Ceci impose de reconnaître, quelles que soient la réalité et les contraintes du moment, que deux voies s'offrent au terminologue dans son activité néologique, celle de la création et celle de l'adoption. Les deux opérations s'appliquent au signe, et non au signifiant, bien qu'elles aient pour objectif l'obtention de ce dernier, pour la seule raison qu'un signifiant n'apparaît jamais seul. Elles ne s'appliquent en aucun cas au signifié, car celui-ci est entre les mains du terminologue bien avant l'engagement de l'activité néologique, dont il est, d'ailleurs, la justification première. De ce fait, les mêmes opérations sont orientées vers le seul signifiant, car, dès que le signe est adopté ou créé, le ou les signifiés qu'il véhicule sont immédiatement écartés, à l'exception bien sûr du signifié qui est en possession du terminologue et qui seul prévaut. Il s'ensuit que, même si c'est le signe qui est créé ou adopté, c'est surtout son signifiant qui est l'objet de l'une ou l'autre des opérations dans l'activité néologique du terminologue.

\section{LA CRÉATION DE SIGNIFIANT}

La création de signifiant impose à l'activité néologique un certain nombre d'étapes. En effet, quelle que soit la connaissance que le terminologue a du signifié à exprimer, le choix de la création d'un signifiant, prenant appui sur une sélection préalable de signes, requiert d'abord une identification rigoureuse de l'organisation interne du signifié en présence, en particulier à travers ses composants sémantiques. Ceux-ci devant, chacun, être exprimés par un signe, la même perspective impose ensuite de repérer, au sein de la langue africaine dans laquelle la terminologie est en élaboration, les signes qui expriment effectivement ces composants.

En fait, ici, dans la pratique du travail, le chercheur effectue un va-et-vient permanent entre l'identification des composants sémantiques du signifié à exprimer, et la recherche des signes dotés de la capacité à véhiculer ceux-ci. Ajoutons que les deux opérations s'influencent mutuellement. L'identification et la connaissance des composants orientera la recherche des signes, les signes en usage dans la langue imposeront un cadre de délimitation des composants.

Enfin, en se soumettant toujours aux modalités requises par la langue africaine, c'est-à-dire aux règles régissant la combinatoire de celle-ci, la création de signifiant imposera d'associer, en un signe nouveau, les signes de la langue qui auront été retenus, le signifiant de ce nouveau signe devant en principe montrer l'aptitude requise pour prendre en charge le signifié à exprimer que le terminologue a en sa possession.

Les procédés de création de signifiant disponibles dans les langues africaines, et dont peut faire usage le terminologue dans le cadre de son activité néologique, sont la composition, la dérivation, la mixation, la conglomération et la syntagmation. 


\section{La composition}

$\mathrm{Si}$, en théorie générale, on veut faire une distinction claire entre les deux procédés de composition et de dérivation, car les langues africaines font usage de formes qui constituent des cas limites, interprétés selon les auteurs comme résultant de l'un ou l'autre de ces procédés, on doit reconnaître que la composition implique l'association de lexèmes seulement, le lexème étant l'unité significative minimale de nature lexicale.

Les langues africaines font largement usage du procédé de composition. Dans la création de signifiant, celui-ci permet l'association d'au moins deux lexèmes, dont résulte une base lexicale qui sera une base composée, simple ou complexe, selon que les lexèmes en présence seront en nombre égal ou supérieur à deux. Si, dans les langues en question, la base composée peut être celle d'un nom ou celle d'un verbe, la composition nominale y est beaucoup plus riche que la composition verbale.

Le procédé de composition révèle des relations différentes entre les lexèmes qu'il associe. Il implique de ce fait sa propre différenciation en trois autres procédés, la composition réduplicative, la complétive et la spécificative.

La composition réduplicative implique le redoublement partiel ou total d'un lexème, dont résulte une base composée réduplicative. Au sein d'une telle formation, apparait, dans le cas général, un lexème associé, soit à une partie, soit à la totalité, de lui-même, c'està-dire, dans ce dernier cas, à un lexème qui lui est identique. Ce cas, qu'on retiendra ici, montre donc l'association par juxtaposition de deux lexèmes identiques. On peut estimer que la relation qui associe ceux-ci est une relation répétitive. Le sens résultant de l'association exprime une répétition de celui véhiculé par chacun des lexèmes. Enfin, l'observation des langues africaines montre que la base lexicale engendrée lors d'une telle association peut être une base nominale ou une base verbale.

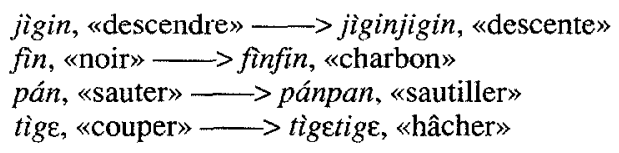

L'interprétation de l'association réduplicative comme résultant d'un procédé de composition n'est pas partagée par tous les auteurs. Certains auteurs 1'interprètent comme résultant d'un procédé de dérivation. Il en est ainsi, pour ne retenir que quelques noms, de Sauvageot (1965: 166-170) pour le wolof, de Hérault (1978: 313-316) pour les faits de redoublement partiel en adioukrou, de Tersis-Surugue (1981: 216-223) pour le zarma, enfin de Dumestre (1987: 299-301) pour le bambara. En fait, si le redoublement partiel, qui s'accompagne souvent de la substitution d'une voyelle étrangère à la voyelle de la première syllabe du lexème, peut être considéré comme un cas limite entre la composition et la dérivation, car il tient de l'une et de l'autre, le redoublement total, quant à lui, semble devoir être interprété comme un fait de composition, car ne faisant usage que de lexèmes (Houis 1981 : 45), et non comme un fait de dérivation, car excluant tout morphème de type dérivatif (Houis 1982: 63).

La composition complétive entraîne l'association, dans le cas le plus simple, de deux lexèmes différents, en une base composée complétive. La relation qui agit ici entre les lexèmes révèle que le signifié de l'un des lexèmes vient compléter celui de l'autre. Le lexème complétant, expansion au sein de la formation, situe, dans l'univers auquel il renvoie, l'univers auquel renvoie le lexème complété, centre de la formation. Selon la langue, l'ordre dans lequel apparaissent les formants révèlera une association progressive ou régressive, selon que le lexème complété sera antéposé ou postposé au lexème complétant. 
Dans les langues africaines, la base lexicale qui est générée ici est surtout une base nominale, mais elle peut aussi être une base verbale.

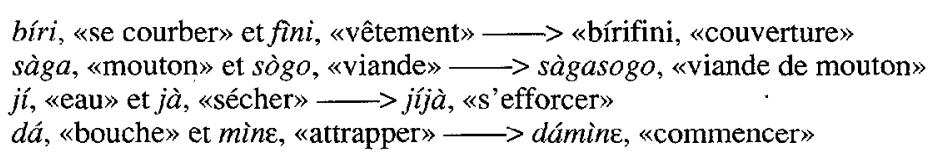

La composition spécificative permet l'association, dans le cas le plus simple, de deux lexèmes différents. Une base composée spécificative résulte de la mise en œuvre de ce procédé. Intervient ici une relation qui implique que le signifié de l'un des lexèmes vient spécifier celui de l'autre. Ainsi, le lexème spécifiant, qui est l'expansion au sein de la formation, exprime un signifié qui apparaît comme un trait du signifié exprimé par le lexème spécifié, lequel est le centre de la formation. Ici aussi, l'ordre d'apparition des formants sera progressif ou régressif, selon que le lexème spécifié sera antéposé ou postposé au lexème spécifiant. La relation d'association qui agit au sein des bases spécificatives se différencie en deux autres relations, l'appositive et la qualificative.

La relation appositive implique, au sein de la base appositive, une autonomie syntaxique entre les formants. Les lexèmes apposé et apposant montrent une autonomie totale, l'un vis-à-vis de l'autre. Ils peuvent, chacun, constituer la base d'une formation qui assumera toute fonction primaire au sein de l'énoncé.

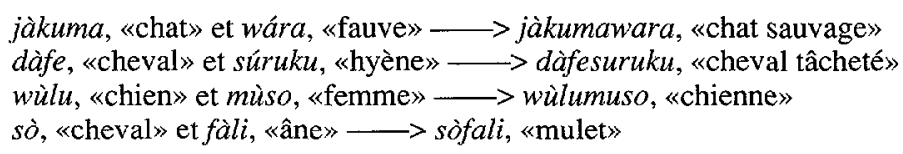

La relation qualificative, quant à elle, montre, au sein de la base qualificative, une dépendance syntaxique. En effet, si le lexème qualifié est, dans le cas général, autonome, le qualifiant est, pour sa part, dépendant de lui. Il ne montre aucune aptitude à constituer la base d'une formation qui assumerait en toute autonomie une fonction primaire au sein de l'énoncé.

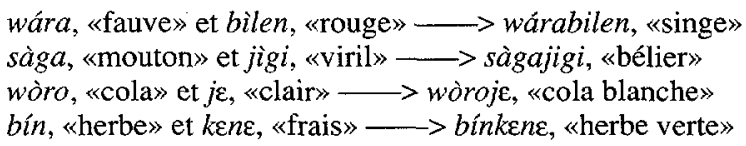

\section{La dérivation}

$\mathrm{Si}$, ici aussi, on veut établir une séparation claire entre les procédés de création de signifiant, on doit admettre que la dérivation implique l'association d'un ou de plusieurs morphèmes dérivatifs, le morphème étant l'unité significative minimale de nature grammaticale, et d'un lexème, et un seul, car plus d'un lexème fonderait l'existence d'une base mixte, tel que présenté plus loin.

Les langues africaines ne font pas toutes le même usage de la dérivation. Il en est, tels le wolof ou le pulaar, qui en font un très large usage, d'autres, tels le bambara ou le soninké, qui en font un usage moyen, et d'autres encore, telles, semble-t-il, les langues kru (Marchese 1983: 98-103), qui en font un usage très restreint. Ces langues offrent des formations dérivées faisant quelquefois usage de plusieurs morphèmes dérivatifs. On conviendra que la base lexicale qui résulte de la mise en œuvre d'un procédé de dérivation sera une base dérivée, simple ou complexe, selon que le nombre des morphèmes dérivatifs en présence sera égal ou supérieur à un. Enfin, sì, dans ces mêmes langues, la 
base dérivée peut être une base nominale ou une base verbale, on doit faire remarquer, tout comme il en a été à propos du procédé de composition, que, dans le cas général, la dérivation nominale y est beaucoup plus riche que la dérivation verbale.

Le morphème dérivatif est l'un des deux éléments qui fondent l'existence de la base dérivée. En effet, si une base lexicale quelconque ne saurait exister sans la présence d'un lexème, une base dérivée ne saurait, quant à elle, exister sans la présence d'un morphème dérivatif. Ce morphème est doté d'un signifiant manifeste, qui peut être tonal, segmental ou tono-segmental. Il occupe une position fixe au sein des bases dans lesquelles il apparait, et il y est le plus souvent suffixé ou préfixé. Enfin, il véhicule toujours une valeur de base, à laquelle peuvent quelquefois s'associer des valeurs de contexte.

Les valeurs véhiculées par les morphèmes dérivatifs aujourd'hui reconnus dans les langues africaines, et qui peuvent servir l'activité néologique du terminologue, sont diverses. On en donne ici un échantillon:

- la valeur diminutive: elle implique une diminution des dimensions exprimées par le lexème ;

- la valeur augmentative: elle implique une augmentation des dimensions exprimées par le lexème;

- la valeur abstractive : elle opère une abstraction à partir du signifié exprimé par le lexème;

- la valeur translative : elle implique une translation d'un état exprimé par le lexème à un autre ; lexème ;

- la valeur réalisative: elle institue l'agent qui réalise l'action exprimée par le

- la valeur instrumentale: elle institue l'instrument qui permet de réaliser l'action exprimée par le lexème ; lexème ;

n la valeur émanative: elle institue l'individu qui émane du lieu signifié par le

- la valeur locative : elle institue le lieu où se trouve l'objet exprimé par le lexème ;

- la valeur attributive : elle implique l'attribution du signifié exprimé par le lexème; lexème ;

- la valeur assimilative: elle implique une assimilation au signifié exprimé par le

- la valeur actitive : elle institue l'acte à partir du signifié exprimé par le lexème ;

- la valeur factitive : elle exprime le faire de l'action signifiée par le lexème;

- la valeur itérative : elle implique une répétition multiple de l'action exprimée par le lexème ;

- la valeur répétitive : elle implique une répétition de l'action exprimée par le lexème;

- la valeur irative : elle exprime 1'aller faire de l'action signifiée par le lexème;

- la valeur vénirative : elle exprime le venir faire de l'action signifiée par le lexème;

- la valeur résultative : elle exprime le résultat de l'action signifiée par le lexème;

- la valeur privative : elle exprime la privation de l'objet signifié par le lexème ;

- lan instrumental

sigi, «asseoir» $\longrightarrow$ sigilan, «chaise»

wúsu, «fumen» - $\longrightarrow$ wúsulan, «encens»

- ba augmentatif

wára, «fauve» - $\longrightarrow$ wáraba, «lion»

mùso, «femme» $\longrightarrow$ mùsoba, «femme de grande notoriété»

- li actitif

tága, «partir» $\longrightarrow$ tágali, «départ»

$f \hat{o}, \ll s a l u e r » \longrightarrow f o l i, \ll$ salutation» 
n la- factitif

bila, «laisser» $\longrightarrow$ lábila, «libérer»

bin, «tomber» $\longrightarrow$ lábin, «faire tomber»

\section{La mixation}

On appelle mixation le procédé de création de signifiant qui associe au sein de la même formation les deux procédés de composition et de dérivation. La base lexicale qui résulte de la mise en application de ce procédé sera appelée base mixte ${ }^{3}$. Dans les langues africaines et, dans le cas le plus simple, la formation de cette base montre, soit une base dérivée et un lexème, associés dans une composition, soit une base composée et un morphème dérivatif, associés dans une dérivation. Il s'ensuit que la mixation peut être de nature compositive ou dérivative. Enfin, le procédé de mixation peut donner naissance à une base nominale ou à une base verbale.

La mixation compositive permet la composition de deux formants dont l'un au moins montre une dérivation. Elle met en cuvre les relations d'association - réduplicative, complétive et spécificative - qui agissent au sein de la composition. Ces relations permettent de distinguer l'une de l'autre les différentes bases mixtes composées.

La base mixte réduplicative montre le redoublement d'une base dérivée. On a donc ici deux formants identiques qui, dans le cas le plus simple, sont chacun constitués d'un lexème et d'un morphème dérivatif.

La base mixte complétive associe, dans une relation dans laquelle l'un complète l'autre, deux formants dont l'un au moins est une base dérivée. On trouve deux schèmes principaux de cette base dans les langues africaines. L'un met en présence un lexème et une base dérivée, dans cet ordre ou dans l'ordre inverse, l'autre associe deux bases dérivées différentes, formées, chacune, d'un lexème et d'un morphème dérivatif.

La base mixte spécificative associe, dans une relation dans laquelle l'un spécifie l'autre, deux formants dont l'un au moins est une base dérivée. On retrouve ici les deux schèmes principaux attestés dans la base mixte complétive.

nyi, «bon» et -man attributif $\longrightarrow$ nyimannyiman, «bon sous tous les angles, très bon»

kála, «arc», dònso, «chasseur» et -ya abstractif $\longrightarrow$ káladonsoya, «chasse à l'arc»

dúmu, «manger», -li actitif et tùma, «temps» $\longrightarrow$ dúmunituma, «moment de manger»

fúran, «balayer», -lan instrumental et kúra, «neuf» $\longrightarrow$ fúralankura, «balai neuf» fò, «saluer», -li actitif et $s \varepsilon b \varepsilon$, «sérieux» $\longrightarrow$ fòlisebe, «salutation honorante»

La mixation dérivative implique la dérivation d'une base déjà composée. Dans le cas le plus simple, la base mixte dérivée montre une base composée et un morphème dérivatif. De ce fait, si une distinction doit être opérée entre les différents types de bases mixtes dérivées, et si cette distinction n'a pas trait aux critères de simplicité et de complexité communs à toutes les bases lexicales ici en question, la distinction ne peut être effectuée qu'à travers les types de bases composées dont peut faire usage la base mixte ici en question. Une telle distinction est effectivement valable dans les langues africaines, où on trouve des bases mixtes dérivées dont la base composée est, soit une base réduplicative, soit une base complétive, soit enfin une base spécificative.

dígi, «appuyer» et -li actitif $\longrightarrow$ dígidigili, «action d'appuyer de manière répétitive» $y \varepsilon l \varepsilon n$, «monter» et -lan instrumental $\longrightarrow$ yelenyelennan, «instrument pour monter de manière répétitive, échelle»

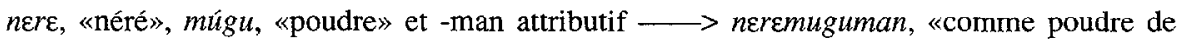
néré» 
$k u ̀ n$, «tête», fहn, «chose» et -to déviatif $\longrightarrow$ kùnfinto, «hystérique»

Ségu, «ville de Ségou», kòro, «vieux» et -ka émanatif $\longrightarrow$ ségukoroka, «habitant du vieux Ségou»

dén, «enfant», misen, «petit» et -ya abstractif $\longrightarrow$ dénmisenya, «enfance»

\section{La conglomération}

Nombreuses sont les langues africaines qui font usage du procédé de conglomération, et, paradoxalement, rares sont les travaux de description de ces langues qui le prennent en compte. Le terme conglomération désigne le procédé de création de signifiant qui opère par délimitation de segments de l'énoncé, puis soudure de la totalité ou d'une partie de ces segments en une base lexicale. Le procédé de conglomération est caractérisé par la classe, le type d'association, l'ordre et le nombre des unités mises en jeu.

Les unités retenues par le procédé de conglomération relèvent du niveau de l'énoncé, s'agissant de noms, de verbes, de morphèmes de syndèse, etc. L'association de ces unités se fera en deux temps. Dans le cas général, les noms et les verbes sont d'abord réduits à leur base. Les bases obtenues et, éventuellement, les morphèmes seront associés par une soudure pure et simple, qui donnera, en un bloc, un nouveau segment, la formation nouvelle. L'ordre qui est ici attesté est celui des formants de l'énoncé, l'ordre syntaxique. Le nombre minimal des unités requises pour une telle construction est de deux, le nombre maximal étant bien sûr celui des éléments de l'énoncé d'origine.

La base lexicale qui résulte de la mise en œuvre du procédé de conglomération est une base conglomérée. Celle-ci est une base nominale. Remarquons que, si le qualifiant «congloméré» est emprunté à Émile Benveniste (1974: 171), la réalité à laquelle il réfère en bambara est quelque peu différente de celle à laquelle il renvoie chez ce linguiste. En effet, contrairement à la conception de celui-ci selon laquelle les unités soudées ne sont ni mutilées ni altérées, ici, elles le sont, en ce sens que, d'un nom ou d'un verbe du niveau de l'énoncé, le procédé ne retient, dans le cas général, seule la base phrastique faisant exception semble-t-il, que la base, les morphèmes associés à ceux-ci étant écartés de la formation conglomérée.

Si on considère la nature des segments de l'énoncé retenus par le procédé, on peut voir trois conglomérations différentes, la phrastique, la rhémique et la rhémo-syndétique.

La conglomération phrastique retient, dans la formation de la future base phrastique, tous les éléments, thème et rhème ${ }^{3}$, d'une phrase donnée. Ceux-ci sont adoptés tels qu'ils apparaissent dans l'énoncé et sont ensuite soudés en une seule et unique base conglomérée, qui exprime le même signifié que la phrase d'origine.

né dâ' té kúma nà, «la bouche de moi n'est pas dans parole» $\longrightarrow$ nédatekumana, «nom d'un quartier de Bamako qui, dans les années cinquante, n'était qu'un hameau éloigné dont les habitants ne pouvaient pas communiquer verbalement avec ceux de la capitale».

bó té né nà, «une sortie n'est pas en moi» $\longrightarrow$ bótenena, «surnom de l'individu de qui on ne peut rien attendre».

La conglomération rhémique puise les formants de la future base rhémique dans le rhème, c'est-à-dire dans le verbe et dans les expansions. Ces dernières sont des nominaux en fonction d'objet ou de circonstant. Le procédé de conglomération retiendra ici la base du verbe et celle du nominal. Il intégrera aussi le morphème postposition, quand celui-ci marque le nominal en fonction de circonstant.

kúma’ nìn bóra né kóno, «parole celle-ci est sortie de moi intérieur» $\longrightarrow$ bó né kóno, «sortir de moi intérieur» $\longrightarrow$ bónekono, «oubli»

ù dára né nà, «ils ont cru moi en» $\longrightarrow$ dá né nà, «croire moi en» $\longrightarrow$ dánena, «confiance» 
La conglomération rhémo-syndétique opère, quant à elle, à partir d'un énoncé faisant usage d'un morphème de syndèse. Celui-ci est un morphème relateur qui attribue à la base verbale qui lui succède au sein de l'énoncé la valeur véhiculée par le morphème verbal en usage dans le verbe qui le précède. Ici aussi, la conglomération puisera les formants de la base conglomérée dans le verbe et les expansions, en fait dans le rhème, mais il s'agit d'un rhème complexe qui contient un morphème de syndèse. Le procédé de conglomération retiendra les bases verbales situées de part et d'autre du morphème de syndèse, le morphème de syndèse lui-même, et, éventuellement, la base du nominal en expansion. Il intégrera, dans le cas de la transitivité de la base verbale qui succède au morphème de syndèse, la base du pronom en fonction d'objet, et dans celui de la présence d'un morphème postposition, ce morphème.

à tágara kà sègin, «il est parti et est revenu» $\longrightarrow$ tága kà ségin, «partir et revenir» $\longrightarrow$ tágakasegin, «va-et-vient».

à yé finin' tà kà à yúguyugu, «il a le vêtement pris et a lui secouer» $\longrightarrow$ tà kà à yúguyugu, «prendre et lui secouer» $\longrightarrow$ tàkaayuguyugu, «frippes».

\section{La syntagmation}

Dans les langues africaines, le syntagme, en tant que formation du niveau de l'énoncé assumant une fonction primaire, peut être un syntagme nominal ou un syntagme verbal. Le syntagme nominal est alors une formation qui associe des noms et/ou des pronoms et/ou des numéraux, etc. Le syntagme de noms, auquel on se limitera ici, devient ainsi la formation qui associe au moins deux noms et qui assume l'une ou l'autre des fonctions imparties aux nominaux. Ici aussi, compte tenu des faits observés, on conviendra que ce syntagme sera simple ou complexe, selon que le nombre des noms dont il fera usage sera égal ou supérieur à deux.

Pris dans sens son large, le concept de syntagmation renvoie au procédé de création de signifiant dont la mise en œuvre donne naissance à un syntagme. Dans le cadre du présent texte, on appellera syntagmation le procédé de création de signifiant qui sélectionne dans la langue deux noms au moins et les associe, selon l'une ou l'autre des relations disponibles, en une formation qui assume toute fonction impartie aux nominaux. On distingue trois types de syntagmation, l'associative, la complétive et la spécificative.

La syntagmation associative unit, dans une association pure et simple, les noms en présence. Elle n'implique aucun apport d'un nom à l'autre, elle laisse à chacun des noms une autonomie totale. La mise en œuvre de ce procédé de syntagmation donne naissance aux syntagmes associatifs.

On peut distinguer, au sein de ce sous-ensemble de syntagmes, les syntagmes réduplicatifs qui manifestent le redoublement d'un nom, des syntagmes distributifs qui révèlent, et un redoublement de nom, et l'intercalation d'un morphème à valeur distributive entre les deux noms identiques, et des syntagmes coordinatifs qui, dans le cas le plus simple, montrent l'intercalation d'un morphème à valeur coordinative entre deux noms qui peuvent être identiques mais qui sont le plus souvent différents.

bòli, «courir, course» - $\longrightarrow$ bòli' bòli', «la course la course, action de courir dans tous les sens» mùso, «femme» $\longrightarrow$ mùso ó mùso, «quelle que soit la femme» màlo, «riz» $\longrightarrow$ màlo' ni màlo', «le riz et le riz»

misi, «bovin» et sàga, «ovin» $\longrightarrow$ misi' ni sàga', «le bovin et l'ovin»

La syntagmation complétive permet d'associer les noms en présence en instituant une relation en vertu de laquelle un nom complète l'autre, ce qui engendre un syntagme 
complétif. Cette relation qui agit au sein du syntagme complétif est identique à celle qui agit au sein de la base complétive présentée dans une section précédente de cet article. Le syntagme complétif, dans le cas le plus simple, montre la présence de deux noms, le complété et le complétant, associés, soit en séquence immédiate, c'est-à-dire par juxtaposition, soit en séquence médiate, dans l'usage d'un morphème à valeur connective intercalé (Houis 1977: 33).

dùgu, «village, terroir» et màsa, «seigneur» $\longrightarrow$ dùgu' màsa', «le seigneur du terroir» mùso, «femme», kà connectif, et filen, «calebasse» $\longrightarrow$ mùso' kà filen', «la calebasse de la femme»

dùgu, «village, terroir», lá connectif et mògo, «homme» $\longrightarrow$ dùgu' lá mògow', «les gens du village»

La syntagmation spécificative institue, entre les noms que sa mise en œuvre permet d'associer, une relation dans laquelle un nom en spécifie un autre. Ce procédé se différencie en deux autres procédés de syntagmation, l'appositif et le qualificatif. Les relations spécificative, appositive et qualificative qui sont en œuvre ici sont identiques à celles qui le sont dans la composition spécificative présentée pécédemment. Concernant les syntagmes qui sont générés ici, le syntagme appositif implique une autonomie des deux noms apposé et apposant en présence, alors que le syntagme qualificatif implique une dépendance du nom qualifiant et, dans le cas général, une autonomie du nom qualifié.

$N c i$, «nom masculin» et fàtok $\varepsilon$, «homme fou» $\longrightarrow N$ ci fàtok $\varepsilon^{\prime}$, «Nci le fou»

$c \varepsilon$, «homme» et kùngwanan, «tête rasée» $\longrightarrow c \varepsilon^{\prime}$ kùngwanan', «l'homme le tête-rasée»

\section{L'ADOPTION DE SIGNIFIANT}

Alors que la voie de la création d'un signifiant impliquait, et le choix de deux signifiants au moins parmi des signifiants connus, et la construction d'un signifiant nouveau par association de ces signifiants, celle de l'adoption d'un signifiant n'implique que le choix d'un signifiant à effectuer parmi des signifiants connus. De même, le processus de l'adoption d'un signifiant ne montre, semble-t-il, que deux étapes essentielles. En effet, si, ici, comme précédemment, le terminologue se doit d'identifier avec rigueur l'organisation du signifié à exprimer en sa possession, il n'est pas tenu d'entrer dans sa composition interne, il n'est pas vraiment tenu d'en connaître les composants sémantiques, le signifié en question devant être pris en charge par un seul signifiant. Par contre, il doit être en mesure de délimiter ce signifié à travers les rapports qu'il entretient avec les autres signifiés de la langue avec lesquels il montre des analogies ou des différences. De plus, il devra rechercher, soit dans la langue africaine dans laquelle la terminologie est en élaboration, soit dans la langue modèle en fonction de laquelle celle-ci l'est, soit, enfin, dans une autre langue africaine en contact, un signifiant appartenant à un signe dont le signifié montre, avec le signifié à exprimer, des rapports tels que le signifiant en question puisse prendre en charge ce dernier signifié.

L'adoption d'un signifiant peut donc être celle d'un signifiant interne ou externe à la langue africaine. Le choix de la recherche de l'un ou l'autre de ces signifiants ne relève pas toujours de la seule volonté du terminologue. Quand le signifié qui doit être exprimé, l'est effectivement dans l'une des langues étrangères mentionnées, le chercheur peut opter pour le signifiant en usage dans cette langue, ou prendre la décision d'ignorer celuici et de rechercher un signifiant en usage dans la langue africaine sur laquelle il travaille. Quand, par contre, le même signifié n'est exprimé dans aucune de ces langues, ne s'offre alors au chercheur qu'une seule possibilité, celle de la recherche d'un signifiant appartenant effectivement à la langue africaine sur laquelle il travaille. Ceci étant dit, il ne faut 
pas perdre de vue la contrainte à laquelle est soumis tout terminologue qui œuvre dans une perspective d'utilisation de la langue, et, donc, de promotion de celle-ci. Dans l'activité terminologique, qui poursuit des objectifs et est soumise à des principes (Halaoui 1991a: 293-300), cette contrainte impose au terminologue d'épuiser, autant que faire se peut, tous les recours possibles à cette langue avant de s'adresser à une langue étrangère. De la prise en compte de cette contrainte dépend largement le développement de la langue.

Le signe dont le signifiant a été retenu en vertu d'un procédé d'adoption est caractérisé par un certain nombre de traits. Le signe à signifiant interne peut montrer une polysémie. Tel est le cas quand le signifiant en question appartenait à un signe qui est en usage dans la langue, ou qui l'était, un nouveau signifié étant attribué à un signifiant qui était déjà associé à un signifié. Cette polysémie peut être un problème au sein d'un langage technique. Ce problème peut cependant être contourné en recourant à une bonne définition terminologique. Le signe à signifiant externe est, quant à lui, monosémique, car le signifié qu'il exprime est nouveau dans la langue, et si, dans sa langue d'origine, son signifiant était associé à plusieurs signifiés, rien ne dit que les utilisateurs de la future terminologie sont au fait de cette polysémie. Enfin, le signe ici en question résulte de la mise en œuvre de l'un ou l'autre des procédés d'adoption de signifiant que sont l'arrogation, pour ce qui concerne le signifiant interne, et l'incorporation, pour ce qui concerne le signifiant externe.

\section{L'arrogation}

La typologie actuelle en matière de néologie française fait une distinction entre la néologie phonologique, la néologie syntagmatique, la néologie sémantique et la néologie d'emprunt (Guilbert 1975: 59). Le mot manifestant une forme et un sens, le néologisme de forme est le mot nouveau qui montre une forme nouvelle, alors que le néologisme de sens est le mot nouveau qui montre un sens nouveau, mais dont la forme est connue dans la langue. Cette forme étant déjà attestée dans la langue, elle y impliquait, avant le fait néologique, un sens qu'elle exprimait. Avec ce fait, elle exprime toujours ce sens, mais, de plus, elle en exprime un autre, celui qui, justement, découle de la pratique néologique. On conclut alors à un enrichissement du sens, donc à un nouveau sens, d'une forme ancienne, on conclut surtout à une création sémantique, à la création d'un sens nouveau (Guilbert 1975 : 69). Le point de vue qui apparaît ainsi ne nous semble pas convenir à la terminologie. Sans vouloir en faire une appréciation détaillée, on dira simplement que ce point de vue est celui du néologue qui étudie les mots nouveaux, les mots nouvellement créés, il n'est pas celui du néologue qui crée les mots, celui du néographe, il n'est pas le point de vue du terminologue qui fait œuvre de néologie.

En effet, ce point de vue implique que le mot nouveau qu'est le néologisme de sens a vu le jour en raison d'une création de sens. Or, il n'en est jamais ainsi pour le terminologue qui crée un mot nouveau. Celui-ci ne crée jamais du sens. Il détient un sens à exprimer, il recherche une forme dans ce but, et il aura un choix à faire quant à la création du mot nouveau, entre les deux possibilités que sont la création d'un signifiant ou l'adoption d'un signifiant. Ni le néologue qui crée les mots ni le terminologue qui fait œuvre de néologie dans cette perspective ne créent du sens. On sait que, pour ce dernier, le sens lui parvient, soit d'une réalité observée et qui n'est pas nommée, soit de la terminologie en langue étrangère en fonction de laquelle il élabore la sienne. Soucieux, dans le cadre du présent travail, de demeurer dans la perspective terminologique, on ne parlera ici ni de néologisme de sens, bien que la réalité à laquelle renvoie cette expression soit celle en cause ici, ni de néologie de sens.

On introduira ici un nouveau concept quant au procédé à présenter, on parlera plutôt d'arrogation, et on définira celle-ci comme le procédé d'adoption de signifiant dans 
lequel le locuteur dans le cas général, ou le terminologue dans le cas présent, utilise, dans le but d'exprimer un signifié qu'il détient, un signifiant de la langue sur laquelle il travaille, un signifiant appartenant déjà à un signe et donc déjà doté d'un signifié. Ce faisant, il attribue, au signifié en sa possession, le signifiant d'un signe qui était ou qui est en usage dans la langue. Du point de vue de la langue dans laquelle la terminologie est en élaboration, on peut dire que le signifié à exprimer s'est arrogé un signifiant, il s'est attribué celui-ci. Du point de vue maintenant de l'homme, le terminologue qui fait œuvre de néographie, on peut dire que celui-ci a, pour reprendre un usage ancien du terme (GLLF 1980), arrogé un signifiant au signifié en sa possession, il a attribué celui-là à celui-ci. Dans les deux cas, le signifiant retenu n'appartenait pas au signifié en question, d'où les actions de s'arroger ou d'arroger, et le terme arrogation ici adopté.

L'arrogation métaphorique est le procédé d'adoption de signifiant à travers lequel le signifiant d'un signe de la langue est utilisé pour exprimer un signifié donné en raison de la similarité manifestée entre ce signifié et celui du signe en question. La similarité, qui apparaît ainsi comme la condition indispensable de la mise en œuvre du procédé ici en question, est observable par le terminologue entre les référents en présence, celui auquel renvoie le signe de la langue dont le signifiant sera arrogé, et celui auquel correspond le signifié à exprimer.

Il n'y a ici aucune création, ni de signifiant ni de signifié. Le terminologue dispose déjà d'un signifié, et il retient un signifiant de la langue qu'il associe en un signe à celuici. On pourra seulement parler de création d'un signe nouveau, car l'association du signifiant et du signifié en question est nouvelle.

Le signe qui résulte de l'association du signifiant arrogé et du signifié à exprimer est une métaphore. Elle est, dans le cas général, une base lexicale. En bambara, cette base peut être une base nominale ou une base verbale.

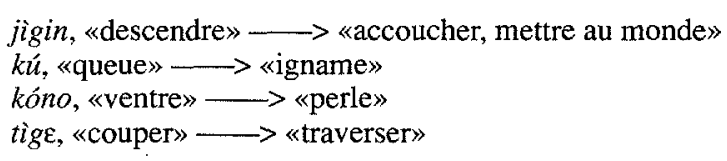

L'arrogation métonymique est le procédé d'adoption de signifiant à travers lequel le signifiant d'un signe de la langue est utilisé pour exprimer un signifié donné en raison de la contiguiité manifestée entre ce signifié et celui du signe en question. Ici aussi, la contiguitté, qui apparaît comme la condition indispensable de la mise en pratique de l'arrogation métonymique, est observable par le terminologue entre les référents en présence.

On pourra dire de même qu'il n'y a ici aucune création, et qu'il y a à la rigueur un signe nouveau en raison d'une association nouvelle d'un signifiant et d'un signifié.

Le signe qui résulte de l'association du signifiant arrogé et du signifié à exprimer est une métonymie. Cette métonymie est, dans le cas général dans les langues africaines, une base nominale.

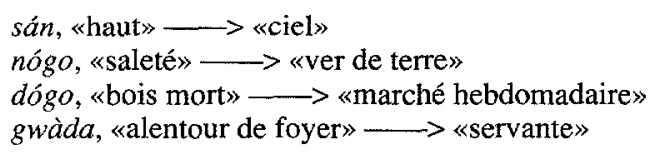

\section{L'incorporation}

Dans la linguistique actuelle, l'emprunt lexical auquel on se limitera ici, est à la fois un procédé et un signe. L'emprunt est d'abord le procédé par lequel une langue incorpore à son vocabulaire, afin d'y être utilisé, un signe lexical appartenant à une autre langue 
dans laquelle il est toujours en usage. L'emprunt est aussi le signe lexical qui est en usage dans une langue donnée, qui y est intégré, car, sans cela, il ne saurait être question d'emprunt (Lacroix 1970: 51-52), mais qui n'appartient pas au fond propre du vocabulaire de celle-ci et qui y a été introduit par un procédé d'emprunt à une autre langue dans laquelle il a été en usage, et peut encore l'être. L'emprunt, 'en tant que procédé, impose ainsi un dédoublement à l'emprunt en tant que signe, car celui-ci appartient à la fois à deux langues.

La notion d'emprunt renvoie dans la néologie d'aujourd'hui à un signe qui montre au moins l'association d'un signifiant et d'un signifié. Un tel constat est fait par le néologue qui étudie les mots nouveaux dans une langue donné. Celui-ci observe dans la langue en étude un mot qui n'appartient pas au fond propre de celle-ci, et qui a été emprunté à une autre langue, au fond propre de laquelle il appartient, ou dans laquelle il est en usage. Le mot en question étant doté d'un signifiant et de l'un au moins des signifiés qu'il véhicule dans cette autre langue, il conclut à la conception de l'emprunt comme étant un signe doté d'un signifiant et d'un signifié. Ce n'est pas le locuteur emprunteur qui accomplit la création... il reçoit cette création comme un fait accompli (Guilbert 1975: 92).

Autre est le point de vue du néologue, qui est aussi celui du terminologue, qui créent les mots nouveaux. Celui-là est en possession d'un signifié qu'il souhaite pouvoir exprimer. Dans ce but, il lui faudra pouvoir attribuer un signifiant à ce signifié. S'il choisit ce signifiant dans une langue étrangère, il fera ce choix à travers, d'une part, l'identification d'un signe de celle-ci qui véhicule le signifié qu'il a en sa possession, d'autre part, l'adoption du seul signifiant de ce signe et l'association à celui-ci du signifié en question, enfin, l'incorporation du signe obtenu à la langue dans laquelle la terminologie est en élaboration. Il s'ensuit que, face à une langue étrangère, le terminologue ne s'approprie pas un signe doté d'un signifiant et d'un signifié, il s'approprie seulement un signifiant.

Par ailleurs, le terme lui-même est difficile à admettre par le terminologue compte tenu du sens que le mot emprunt véhicule dans la langue générale. En effet, en terminologie, le terme emprunté, qui constitue une solution à un problème de communication, en venant combler une lacune manifestée par la langue dans sa prise en charge de la communication dans une spécialité donnée, est un élément constitutif d'un système, dont il ne saurait être séparé, sous peine d'entraîner des déséquilibres au sein de celui-ci. Pour le terminologue, il n'y a pas seulement emprunt, car la notion d'emprunt peut renvoyer à une prise de possession momentanée, il y a bel et bien incorporation.

On parlera donc ici d'incorporation pour désigner le procédé d'adoption de signifiant par lequel le locuteur utilise, dans le but d'exprimer un signifié en sa possession, un signifiant appartenant à une autre langue dans laquelle il exprime, entre autres signifiés éventuels, le signifié en question. Ce faisant, il incorpore à sa langue un signe dont le signifiant n'appartenait pas à celle-ci. Au terme du processus d'incorporation, la base obtenue peut être appelée base incorporée. Au cours de son incorporation, afin de devenir, du signifiant d'une langue étrangère qu'il était, un signifiant de la langue qui incorpore, celui-ci pourra connaître un certain nombre de modifications qui seront constitutives de ce qu'il est convenu d'appeler l'intégration. Les deux langues en présence pouvant montrer des différences, dans leurs structures respectives, l'intégration du signifiant à incorporer révèlera, en particulier, une adaptation aux structures de la langue qui le reçoit. De ce fait, l'importance des modifications de notre signifiant sera, en principe, proportionnelle à l'importance des différences reconnues entre les deux langues.

On retiendra ici le passage du français au bambara et on donnera des exemples d'intégration phonologique et d'intégration morphologique. 
- changement de consonne $\mathrm{v} \longrightarrow \mathrm{w}$

«valise» $\longrightarrow$ wàlisi
«voiture» $\longrightarrow$ wàtiri
«volant» $\longrightarrow$ wàlan
«Sauvage» $\longrightarrow$ sówasi

- intégration dans une base composée

bàlon, «ballon» et tán, «donner un coup de pied» $\longrightarrow$ bàlontan, «football»

bònbon, «bonbon» et kàla, «tige» - bònbonkala, «sucette»

- formation d'une base dérivée en -ya abstractif

pèresidan, «président» $\longrightarrow$ pèresidanya, «êtat/condition de président»

minise, «menuisier» $\longrightarrow$ miniseya, «état/condition de menuisier»

\section{Notes}

1. Pour chacune de ces langues, sont donnés ci-dessous le groupe auquel elle appartient et le pays où est en usage le parler concerné.

l'adioukrou: kwa, Côte d'Ivoire, le bambara: mandé, Mali, le banda-linda: oubanguien, Centrafrique, le baoulé : kwa, Côte d'Ivoire, le ciluba : bantou, Zaïre, le dioula : mandé, Côte d'Ivoire, le kasim : gur, Burkina Faso, le kirundi : bantou, Burundi, le mina : kwa, Bénin Togo, le mandinka : mandé, Gambie, le niwoli : kru, Côte d'Ivoire, le nugunu : bantou, Cameroun, le peul : ouest-atlantique, Cameroun, le peul pulaar: ouestatlantique, Sénégal, le podoko : tchadique, Cameroun, le san : mandé, Burkina Faso, le sango : oubanguien, Centrafrique, le soninké : mandé, Mauritanie, le wolof : ouest-atlantique, Sénégal, le zarma : songhay-zarma, Niger

2. La connaissance du bambara qui est donnée ici provient, pour la composition, la dérivation, la conglomération et la syntagmation, de Dumestre (1987) et des propres recherches de l'auteur, et de ces dernières, pour le reste.

Le bambara est une langue à tons, ceux-ci apparaissent aux deux niveaux lexical et grammatical. L'accent aigu, l'accent grave et l'apostrophe représentent, respectivement, le ton lexical haut, le ton lexical bas et la valeur grammaticale du défini.

3. L'auteur remercie Dafrassi Sanou, pour lui avoir suggéré le qualifiant «mixte» pour nommer la base mixte, et André Clas, pour lui avoir rappelé l'opposition «thème / rhème» qui a été utilisée dans la dénomination des bases conglomérées.

NAZAM HALAOUI

Université de Montrêal, Montréal, Canada

\section{RÉFÉRENCES}

BENVENISTE, Émile (1974): Problèmes de linguistique générale 2., Paris, Gallimatd.

DUMESTRE, Gérard (1987) : Le bambara du Mali. Essais de description linguistique, Paris, Université Paris III.

FODOR, István et Claude HAGĖGE (dir.) (1983, 1983, 1984): La réforme des langues. Histoire et avenir, Hambourg, Helmut Buske Verlag Hamburg, 3 vol. (Contributions de Andrzejewski sur le somali, de Coupez sur la langue rwandaise, de Dumont et Mbodj sur le wolof, de Jungraithmayr sur le hausa, de Louw sur le xhosa et le zulu, de Mosha sur le luganda, de Polomé sur le swahili, de Rodegem sur le rundi, de Samarin et Diki-Kidiri sur le sango, et de Tubiana sur l'amharique.)

Grand Larousse de la langue française (1980): Paris, Larousse.

GUILBERT, Louis (1975) : La créativité lexicale, Paris, Larousse.

GUIRAUD, Pierre (1967): Structures étymologiques du lexique français, Paris, Larousse.

HALAOUI, Nazam (1989): Questions de méthode en terminologie des langues africaines, Paris, ACCT.

HALAOUI, Nazam (1991a) : «La terminologie des langues africaines. Esquisse d'une problématique», Meta, 36-1, pp. 291-300.

HALAOUI, Nazam (1991b) : «De l'acte préparatoire à l'enquête terminologique», Terminogramme, 59, pp. 4-8.

HALAOUI, Nazam (1991c) : «Terminologie, traduction et développement», Terminologies nouvelles, 6, pp. 44-47.

HÉRAULT, Georges (1978) : Éléments de grammaire adioukrou, Abidjan, ILA.

HOUIS, Maurice (1977): «Plan de description systématique des langues négro-africaines», Afrique et langage, 7, pp. 5-65. 
HOUIS, Maurice (1981): «La relation de détermination en syntagmes et composés nominaux», Afrique et langage, 16, pp. 5-47.

HOUIS, Maurice (1982): «De la dérivation à travers quelques langues africaines», Modèles linguistiques, 4-2, pp. 49-67.

LACROTX, Pierre-Francis (1970): «Cultures et langues africaines: les emprunts linguistiques», Langages, 18, pp. 48-64.

MARCHESE, Lynel (1983): Atlas linguistique kru, Abidjan, ILA-ACCT.

SAUVAGEOT, Serge (1965) : Description synchronique d' un dialecte wolof : le parler du Dyolof, Dakar, IFAN. TCHITCHI, Toussaint Yaovi (1990): «Terminologie et transfert de technologie», Cahiers d'études linguistiques, 4-5, pp. 2-135.

TERA, Kalilou et Siaka TOURE (1983): Propositions pour la création d'un vocabulaire scientifique en jula, Abidjan, ACCT-ILA.

TERSIS-SURUGUE, Nicole (1981): Économie d' un système. Unités et relations syntaxiques en zarma (Niger), Paris, SELAF-ACCT. 